\title{
Coupling and power transfer efficiency enhancement of modular and array of planar coils using in-plane ring-shaped inner ferrites for inductive heating applications
}

V. T. Kilic, E. Unal, and H. V. Demir

Citation: Journal of Applied Physics 122, 014902 (2017); doi: 10.1063/1.4992119

View online: https://doi.org/10.1063/1.4992119

View Table of Contents: http://aip.scitation.org/toc/jap/122/1

Published by the American Institute of Physics

\section{Articles you may be interested in}

Characterisation of elastic turbulence in a serpentine micro-channel

Physics of Fluids 29, 083102 (2017); 10.1063/1.4996356

Graphene and related two-dimensional materials: Structure-property relationships for electronics and optoelectronics

Applied Physics Reviews 4, 021306 (2017); 10.1063/1.4983646

Indonesian commercial bus drum brake system temperature model

AIP Conference Proceedings 1717, 050003 (2016); 10.1063/1.4943478

Frequency dependence of sound speed and attenuation in marine sediments

The Journal of the Acoustical Society of America 120, 3099 (2006); 10.1121/1.4787535

Comparisons of Biot and grain-shearing theories: sound speed, attenuation and acoustic impedance

The Journal of the Acoustical Society of America 123, 3350 (2008); 10.1121/1.2933913

Artificial neural network intelligent method for prediction

AIP Conference Proceedings 1872, 020021 (2017); 10.1063/1.4996678

\section{PHYSICS TODAY}

MANAGER'S GUIDE

WHITEPAPERS

\section{READ NOW}

PRESENTED BY

Accelerate R\&D with
Multiphysics Simulation 


\title{
Coupling and power transfer efficiency enhancement of modular and array of planar coils using in-plane ring-shaped inner ferrites for inductive heating applications
}

\author{
V. T. Kilic, ${ }^{1, a)}$ E. Unal, ${ }^{1}$ and H. V. Demir ${ }^{1,2}$ \\ ${ }^{1}$ Department of Electrical and Electronics Engineering, Department of Physics, and UNAM - Institute of \\ Materials Science and Nanotechnology, Bilkent University, Ankara 06800, Turkey \\ ${ }^{2}$ School of Electrical and Electronic Engineering, School of Physical and Mathematical Sciences, \\ Nanyang Technological University, Singapore 117602
}

(Received 12 April 2017; accepted 26 June 2017; published online 7 July 2017)

\begin{abstract}
We propose and demonstrate a highly effective method of enhancing coupling and power transfer efficiency in inductive heating systems composed of planar coils. The proposed method is based on locating ring-shaped ferrites in the inner side of the coils in the same plane. Measurement results of simple inductive heating systems constructed with either a single or a pair of conventional circular coils show that, with the in-plane inner ferrites, the total dissipated power of the system is increased by over $65 \%$. Also, with three-dimensional full electromagnetic solutions, it is found that power transfer efficiency of the system is increased up to $92 \%$ with the inner ferrite placement. The proposed method is promising to be used for efficiency enhancement in inductive heating applications, especially in all-surface induction hobs. Published by AIP Publishing.

[http://dx.doi.org/10.1063/1.4992119]
\end{abstract}

\section{INTRODUCTION}

Far-field electromagnetics has been studied for decades. Its theory is well known and widely used in numerous applications, including communication and radar technologies. Similarly, electromagnetic fields in the near-field have been fully understood and day after day its application areas grow. For example, wireless induction utilizes electromagnetic fields in the near-field. Today, induction systems are being used in a wide range of areas including heating and energy transfer. ${ }^{1-6}$ In addition to the widespread use of induction heaters in heavy industries such as metal melting, induction hobs have become increasingly more popular in recent times because of its advantages such as safety, cleanliness, quick warming, and controllability. 5,6

In induction systems, energy transfer is achieved via induced eddy currents on the receiver coil. These eddy currents are in reverse directions with those of the currents in the transmitter coil and are induced by coupling of the produced magnetic flux. In inductive heating, instead of a receiver coil, there exists a vessel. Magnetic fluxes, produced by the coils beneath the hob surface, couple to the vessel, and eddy currents are induced on it. Because of the vessel's self-resistance, eddy currents generate heat and warm up the vessel. Previously, coils of various shapes, such as circular and elliptical ones, were developed for efficient inductive heating. ${ }^{7,8}$ In addition, in newly designed hobs, an array of small-sized coils with different shapes are used for all-surface efficient inductive heating. ${ }^{9-12}$ Using an array of small-sized coils, it is possible to control field localization and orientation and to power on only the specific coils that are covered by the vessel in predetermined

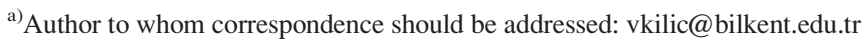

proportions. ${ }^{13}$ This is indeed similar to the array antenna theory that enables efficient power transmission to receiver coils where the receiver coil is a vessel, and it is in the nearfield here.

In regular induction hobs, efficiency enhancement is obtained with ferrites located below the coils. ${ }^{14-16}$ However, this enhancement is limited, and further improvements are needed. On the other hand, in some designs, ferrites of different shapes, such as U-shaped ferrites, were placed around coils and/or coil windings. ${ }^{17-19}$ Although such assemblies increase heating in conventional systems by focusing magnetic flux, they inhibit coupling between side-by-side placed coils in all-surface induction hobs. In an array structure, coupling between coils is an important factor for the vessel's efficient heating, which was investigated in the literature both analytically and experimentally. ${ }^{20,21}$ Therefore, it is necessary to develop new methods to improve the efficiency of inductive heating systems without prohibiting coupling between coils in array forms.

Different from the previous studies, here, we propose and demonstrate coupling and power transfer efficiency enhancement in an inductive heating system with ferrites located in the inner side of the side-by-side placed coils in the same plane. This is intended especially for array structures used in all-surface heating application. The proposed method relies on decreasing the volume in which the produced magnetic flux is localized and increasing the flux density. The inner ferrites achieve this by redirecting magnetic fields, and the resultant high density magnetic flux enables increased coupling in the system and enhancement in the power transfer efficiency. Meanwhile, the inner ferrites do not decrease magnetic flux generation of the coils because magnetic fields are produced from the winding region of the coils. 


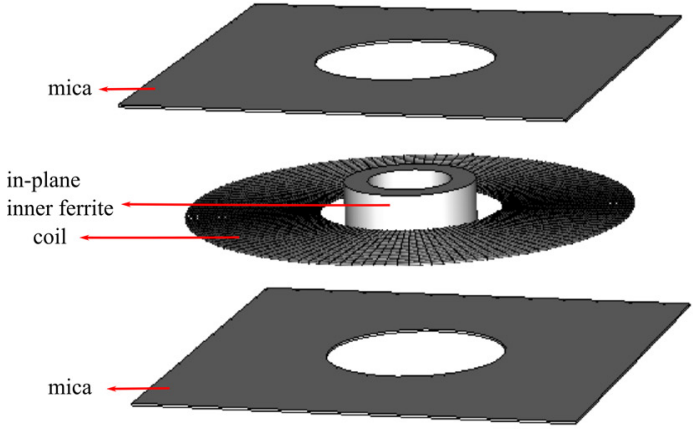

(a)

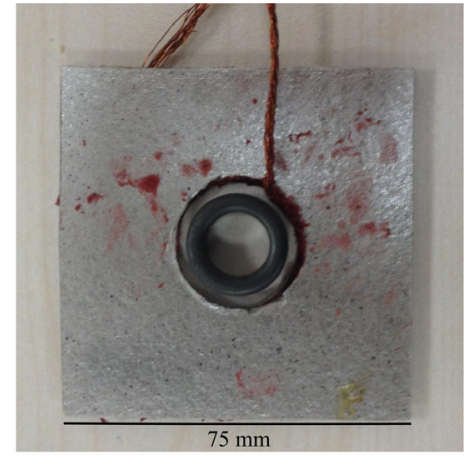

(b)
FIG. 1. (a) Coil structure used in measurements and ring-shaped ferrite located in the inner side of the coil (where mica layers and the circular coil are illustrated to be apart from each other for clear visibility, although they are in contact in the actual structure). (b) Photograph of the produced coil from top view together with ringshaped ferrite placed inside the coil.

\section{EXPERIMENT}

\section{A. Single coil arrangement}

We initially examined power transfer efficiency and coupling enhancements in an inductive heating system composed of a single coil unit with inner ferrite located inside the coil. To this end, measurements were obtained systematically, i.e., with and without loading, and by adding inner ferrite.

\section{Without loading}

The coil structure and a photograph of one of the produced coils used in measurements are shown in Fig. 1 together with a ring-shaped inner ferrite. Here, the circular coil is sandwiched between two mica layers. Each mica has $0.4 \mathrm{~mm}$ thickness and is used as a substrate layer during production. Each coil has an inner diameter of $30 \mathrm{~mm}$ and an outer diameter of $75 \mathrm{~mm}$. Also, they have 44 turns in total, where each turn is composed of 13 copper wires with $0.25 \mathrm{~mm}$ diameter.

The measurements were done at $20 \mathrm{kHz}$ and $100 \mathrm{kHz}$ because conventional induction heaters usually operate between these frequencies. The inductance of the produced coils without a ring-shaped inner ferrite was measured to be $102.65 \mu \mathrm{H}$ at $20 \mathrm{kHz}$ and $102.03 \mu \mathrm{H}$ at $100 \mathrm{kHz}$. Similarly, the self-resistance of the coils was measured $324.75 \mathrm{~m} \Omega$ at $20 \mathrm{kHz}$ and $914.75 \mathrm{~m} \Omega$ at $100 \mathrm{kHz}$.

We repeated our inductance and resistance measurements of the produced coils with a Mn-Zn ferrite layer that is placed just below the coils and has $7 \mathrm{~mm}$ thickness. Locating a ferrite layer below the coils is a well-known method to increase coupling and efficiency of inductive heating systems. ${ }^{14-16}$ This is explained by the image theory, i.e., magnetic fields produced by the coils in bottom half space is redirected upwards by the ferrite layer. The inductance and the resistance values of the coils were measured to be $170.97 \mu \mathrm{H}$ and $520.00 \mathrm{~m} \Omega$ at $20 \mathrm{kHz}$ and $169.42 \mu \mathrm{H}$ and 1.65 $\Omega$ at $100 \mathrm{kHz}$, respectively. The enhancement in the resistance value is unwanted because it indicates large power loss in the system. However, the increase in the inductance value represents coupling enhancement with a denser magnetic flux in the inductive heating system.

\section{With loading}

Next, we placed a loading plate above the coil and investigated changes in inductance and resistance values. The loading plate was selected to be made of a stainless steel (AISI 430) having a cylindrical shape with $180 \mathrm{~mm}$ diameter and $1 \mathrm{~mm}$ thickness because stainless steel is one of the most appropriate material types to be used in inductive heating applications. In addition, since the steel plate is used to imitate a vessel, its diameter is selected to be typical for conventional vessel diameters. The constructed system is shown in Fig. 2 from its top and cross-sectional side views.

In the system, the distance between the coil plane and the steel plate $\left(h_{1}\right)$ is set to $10 \mathrm{~mm}$, similar to that in conventional hobs (using vitro ceramic glass). Moreover, in the figure, the ring-shaped ferrite in the inner side of the coil is

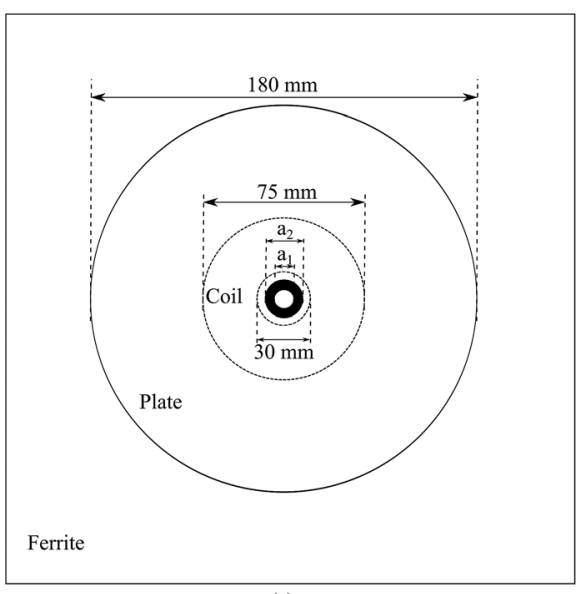

(a)

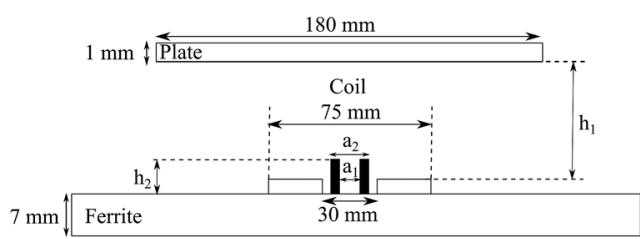

FIG. 2. Schematic of the constructed setup with a single circular coil from (a) top and (b) cross-sectional side views. 
marked in black. It has a height of $9 \mathrm{~mm}\left(\mathrm{~h}_{2}\right)$, an inner diameter of $14 \mathrm{~mm}\left(a_{1}\right)$, and an outer diameter of $22 \mathrm{~mm}\left(a_{2}\right)$. Similar to the ferrite layer below the coils, the ring-shaped ferrites are also made of Mn-Zn. Ferrites of this geometry and material type are commonly cheap and commercially available. Also, it is important to note that $4 \mathrm{~mm}$ wall thickness is higher than the ferrite material's skin depth at specified frequencies.

Measurements were conducted with and without the ring-shaped inner ferrite. Inductance and resistance values of the loaded coil in the system without the inner ferrite were measured as $167.05 \mu \mathrm{H}$ and $4.08 \Omega$ at $20 \mathrm{kHz}$ and $139.79 \mu \mathrm{H}$ and $13.58 \Omega$ at $100 \mathrm{kHz}$, respectively. The decrease in the equivalent inductance values is because of induced eddy currents on the loading steel plate. Based on electromagnetic law of induction, eddy currents are induced on the plate that is in opposite direction with the currents in the coil to decrease change in magnetic flux produced by the coil. A high decrease in the inductance at $100 \mathrm{kHz}$ frequency indicates strong coupling between the plate and the coil. On the other hand, resistance values increase too much in the system with respect to those measured before in the system without the loading plate. The increase in the measured resistance values is due to the fact that in addition to self-heating losses in the coil and the below ferrite layer, part of the input power is transferred to and dissipated on the plate as heat.

Moreover, for comparison purposes, measurements were repeated in the system with the ring shape ferrite placed inside the coil. The system is exactly the same with the setup represented in Fig. 2. Equivalent inductance and resistance values of the coil were found to be $210.35 \mu \mathrm{H}$ and $6.91 \Omega$ at $20 \mathrm{kHz}$ and $166.53 \mu \mathrm{H}$ and $20.84 \Omega$ at $100 \mathrm{kHz}$, respectively. Results show that in the constructed system, the coil's equivalent inductance increases approximately $26 \%[(210.35 \mu \mathrm{H}-167.05 \mu \mathrm{H}) / 167.05 \mu \mathrm{H}]$ at $20 \mathrm{kHz}$ and $19 \%[(166.53 \mu \mathrm{H}-139.79 \mu \mathrm{H}) / 139.79 \mu \mathrm{H}]$ at $100 \mathrm{kHz}$. Enhancement in the equivalent inductance indicates a high magnetic flux density and a strong coupling. Similarly, the equivalent resistance of the coil increases around $69 \%$ $[(6.91 \Omega-4.08 \Omega) / 4.08 \Omega]$ at $20 \mathrm{kHz}$ and $53 \%$ [(20.84 $\Omega$ - $13.58 \Omega) / 13.58 \Omega$ ] at $100 \mathrm{kHz}$. The system's equivalent resistance represents total dissipated power in the system based on the following relation:

$$
P_{\text {total }}=I^{2} \times R_{e q},
$$

where $P_{\text {total }}$ is the total dissipated power, $I$ is the supplied current, and $R_{e q}$ is the equivalent resistance. Therefore, enhancement in the system's equivalent resistance with the placement of the ring-shaped ferrite denotes considerable amount of increase in the total dissipated power in the system. In other words, with the same supply currents to the coil, the total dissipated power in the system is increased more than its half with the ferrite placement inside the coil.

To determine how much of the increase in resistance is due to the power transfer efficiency enhancement and how much of it is because of the increase in coil winding losses and ferrites' self-heating, we modeled and simulated the system shown in Fig. 2 with three-dimensional (3D) electromagnetic solver. Simulations were repeated with and without the ring shape ferrite inside the coil. The constructed system model in the simulations is exactly the same as the setup represented in Fig. 2, except the mica layers below and top of the coil were modeled to be air regions because mica and air have similar effects on system's electromagnetic properties. Therefore, in the simulated system, $0.4 \mathrm{~mm}$ gap exists between the bottom ferrite layer and the coil windings. On the other hand, in the simulations, the ring-shaped inner ferrite is in contact with the bottom ferrite layer as in the measurement setup.

In the modeled system, the plate's material was selected to be AISI 430 that has relative permittivity $\left(\varepsilon_{\mathrm{r}}\right)$ equals to 1 and relative permeability $\left(\mu_{\mathrm{r}}\right)$ equals to 950 . Also, electrical conductivity $(\sigma)$ of AISI 430 was set to $1.67 \times 10^{6}(\mathrm{~S} / \mathrm{m})$. Besides, the coil was modeled to be made of copper $(\mathrm{Cu})$ material. Since manufactured coils consist of several litz strands, the simulations current was forced to flow on entire cross section of the coil windings. Moreover, the ring-shaped inner ferrite and the bottom ferrite layer were modeled to be made of $\mathrm{Mn}-\mathrm{Zn}$ with relative permittivity $\left(\varepsilon_{\mathrm{r}}\right)$ and relative permeability $\left(\mu_{\mathrm{r}}\right)$ equal to 270 and 640 , respectively. Eelectrical resistivity $(\rho)$ of the $\mathrm{Mn}-\mathrm{Zn}$ was set to $6.5(\Omega \mathrm{m})$. High permeability of the plate material yields enhanced coupling of the magnetic flux produced by the coil to the plate. In addition, its electrical conductivity, which is lower than that of the gold, copper, aluminum, or similar metals, causes quick warming by converting the induced eddy currents into heat. Therefore, electrical and magnetic characteristics of the plate material enable efficient power transmission together with large variations in the equivalent inductance and resistance values. ${ }^{13}$ Similarly, ferrites with very high permeability are important for system efficiency enhancement and increase in equivalent inductance and resistance values of the coil because ferrite's effect is proportional with its magnetic permeability. ${ }^{14}$

In the simulations, it is found that in both cases, losses on the ferrites are considerably small with respect to other power dissipations, i.e., less than $1 \%$ of the power is lost on the ferrites as self-heating. In addition, coil winding losses were calculated to not increase too much with the inner ferrite's placement. On the other hand, power transfer efficiency of the system increases from $88 \%$ to $91 \%$ at $20 \mathrm{kHz}$ and from $86 \%$ to $89 \%$ at $100 \mathrm{kHz}$ with the ring-shaped ferrite's placement. Power transfer efficiency is ratio of the power that is transferred to and dissipated on the plate to total power dissipated in the system, which includes the transferred power and all the losses in the system. Power transfer efficiency of the system is calculated with Eq. (2)

$$
\text { Efficiency }=100 \times \frac{P_{\text {transferred }}}{P_{\text {total }}},
$$

where Efficiency is the power transfer efficiency of the system in percentages. Also, $P_{\text {transferred }}$ is the transferred power to the plate, and $P_{\text {total }}$ is the total dissipated power in the system.

Moreover, for comparison purposes, we calculated inductance values in the system. Similar results with those obtained in the measurements were found. For instance, in 
the simulated system where ring-shaped ferrite is not placed inside the coil, the equivalent inductance of the coil was calculated to be $165.30 \mu \mathrm{H}$ at $20 \mathrm{kHz}$ and $127.01 \mu \mathrm{H}$ at 100 $\mathrm{kHz}$. However, for the same system, the equivalent inductance of the coil was measured as $167.05 \mu \mathrm{H}$ at $20 \mathrm{kHz}$ and $139.79 \mu \mathrm{H}$ at $100 \mathrm{kHz}$.

\section{B. Double coil arrangement}

The system was further analyzed with a pair of circular coils instead of a single one. The new system helps to understand the coupling and efficiency changes in an all-surface inductive heating system with placement of the ferrites inside the coils. Experiments were repeated systematically in the new system with and without loading.

\section{Without loading}

To investigate changes in coils coupling, two circular coils were placed side by side on a ferrite layer and electrically connected in series. The ferrite layer is the same with that used in previous measurements, which was shown in Fig. 2. The total inductance and resistance of the coils were measured for different coil distances. Results are shown in Fig. 3. Here, $0^{\circ}$ and $180^{\circ}$ phase differences indicate that the currents flowing on the coils are in the same direction (e.g., both in clockwise) and in reverse directions (one coil in clockwise and the other coil in counterclockwise), respectively. In Figs. 3(a) and 3(c), as the coil separation increases, inductances in $0^{\circ}$ and $180^{\circ}$ phase difference cases get closer to each other and converge to values, which are marked with black lines. These are equal to the sum of individual inductances, i.e., two times the inductance of the coil on the ferrite plane (since the coils are identical). This convergence over distance indicates that coupling decreases between the coils. On the other hand, low and high inductance values obtained with the $0^{\circ}$ and $180^{\circ}$ phase differences are because of the destructive and constructive coupling of the coils, respectively. ${ }^{22}$ Similarly, in Fig. 3(d), resistances in $0^{\circ}$ and $180^{\circ}$ phase difference cases converge to a value, which is two times the resistance of the coil on the ferrite plane and pointed out with a black line. However, in Fig. 3(b), there is no such a convergence. The total resistance value of the systems is close to the double of the resistance of the coil on the ferrite plane, which is again marked with a black line. Since the resistance of the coils is low, the variation in the system resistance with the distance is small, too, i.e., in the order of tens of $\mathrm{m} \Omega$. The indecisive changes in the resistance values at $20 \mathrm{kHz}$ frequency might be due to precision of the measurement.

\section{With loading}

Next, we placed the steel plate that is exactly the same with the plate used in single coil arrangement above the coils. Schematic of the new system is illustrated in Fig. 4. Different from the system represented in Fig. 2, here, the setup contains two side-by-side placed circular coils connected in series with a distance d between them. On the other hand, all the parameters in the system are the same with those in Fig. 2. For instance, ring-shaped inner ferrites have a height of $9 \mathrm{~mm}\left(\mathrm{~h}_{2}\right)$, an inner diameter of $14 \mathrm{~mm}\left(\mathrm{a}_{1}\right)$, and an outer diameter of $22 \mathrm{~mm}\left(\mathrm{a}_{2}\right)$. These ferrites inside the coils are again marked in black. Also, the distance between the steel plate and the coils' top surface $\left(\mathrm{h}_{1}\right)$ is $10 \mathrm{~mm}$.

We obtained measurements with and without the ringshaped inner ferrites. As represented in Fig. 1, ring-shaped ferrites are placed inside the coils such that their centers coincide with the coils' centers to further enhance coupling in the system. Measured inductance and resistance values of the systems are shown in Fig. 5 for different coil distances. Here, the maximum distance between the coils is set to $3 \mathrm{~cm}$ because the coils start to overflow from the plate's projection.

In the figure, similar behavior to Fig. 3 is observed. Since $180^{\circ}$ phase difference exists between the coils, the inductance and the resistance values decrease with the distance. These reductions in the $180^{\circ}$ phase difference application are because of the constructive coupling between the coils. In addition, the inductance values shown in Figs. 5(a) and 5(b) are lower than the inductance values illustrated in Figs. 3(a) and 3(c), respectively. The plate decreases the
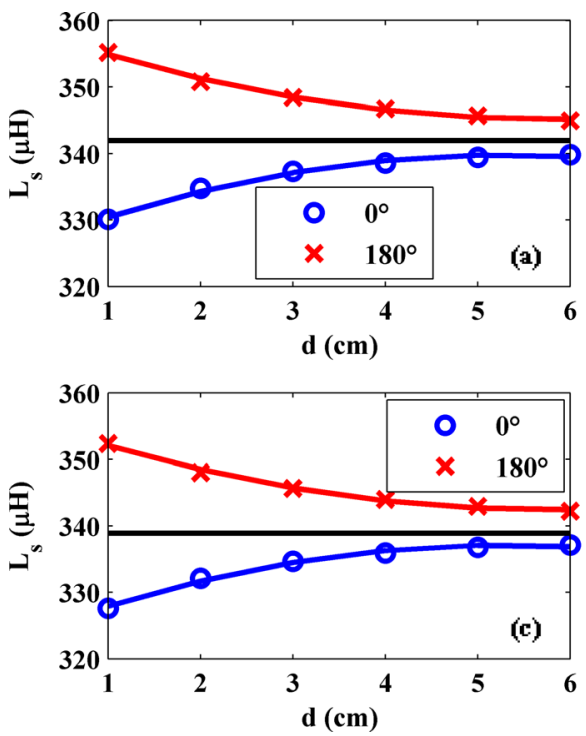
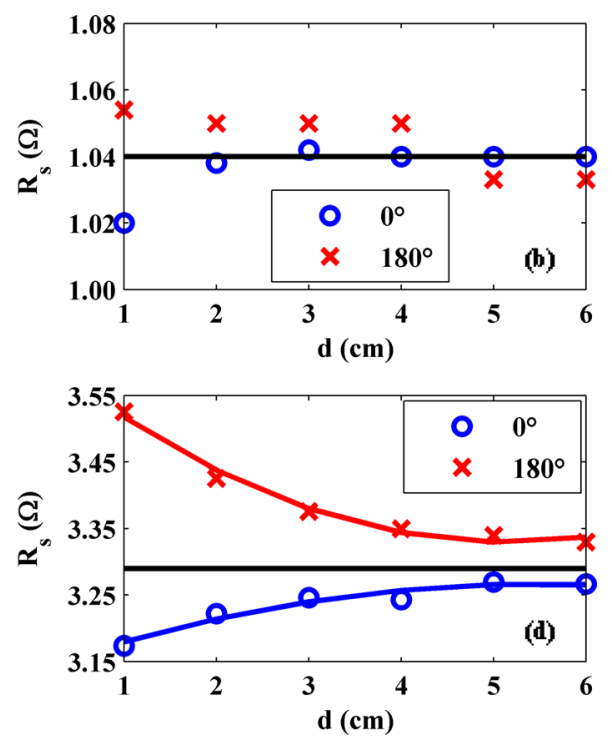

FIG. 3. Inductance and resistance change in the system that is composed of two side-by-side placed and series connected coils for various distances between the coils with $0^{\circ}$ and $180^{\circ}$ phase differences at (a), (b) $20 \mathrm{kHz}$ and (c), (d) $100 \mathrm{kHz}$. In parts (a), (c) and (d), red and blue curves represent the second order fittings. [In part (b), in order not to make the plot more complex, fitting curves are not presented.]. 


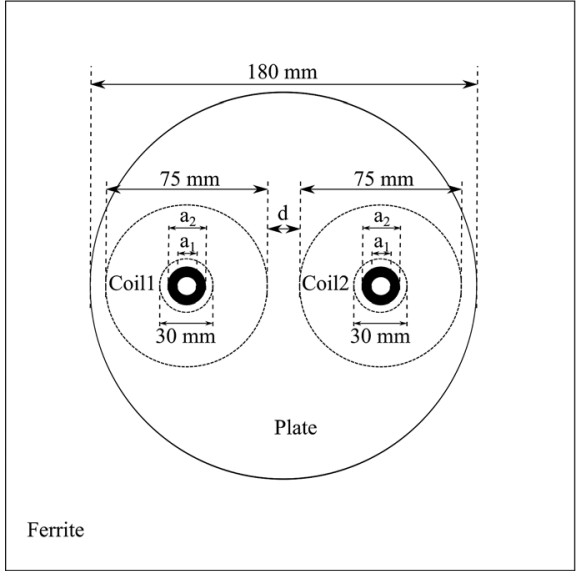

(a)

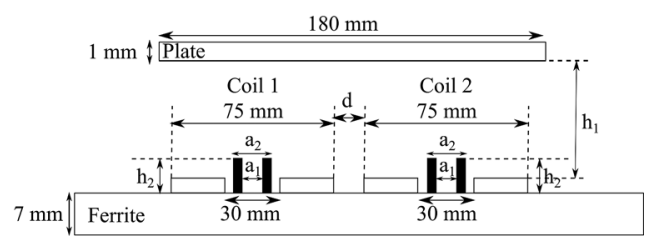

(b)
FIG. 4. Schematic of the constructed setup with a double circular coil from (a) top and (b) cross-sectional side views. system's equivalent inductance via induced eddy currents on it. On the other hand, the resistance values presented in Figs. 5(a) and 5(b) are considerably higher than those shown in Figs. 3(b) and 3(d), respectively. Therefore, with the plate's placement total power dissipated in the system increases. With numerical simulations of the system, it was demonstrated that the enhancement in resistance is mostly because of the power transferred to and dissipated on the plate.

Also in Fig. 5, the equivalent inductances decrease as the frequency increases, while the equivalent resistances in the system increase. The increase in the equivalent resistance is mostly because of the enhancement in the plate's selfresistance that is due to the decrease in the skin depth of the plate's material. On the other hand, the decrease in the equivalent inductance indicates coupling increase between the plate and the coils. Since the plate couples to the coils more, eddy currents with higher magnitudes are induced on it, which decreases the system's equivalent inductance.

Moreover, comparisons of Fig. 5(a) with Fig. 5(c) and Fig. 5(b) with Fig. 5(d) show that with placements of ringshaped inner ferrites, the system's equivalent inductance and resistance values increase considerably. These enhancements are calculated using Eqs. (3) and (4), and they are shown in Fig. 6

$$
\begin{aligned}
& \Delta L=100 \times\left[\left(L_{w_{-} \text {ferrite }}-L_{w / o_{-} \text {ferrite }}\right) / L_{w / o_{-} \text {ferrite }}\right] \\
& \Delta R=100 \times\left[\left(R_{w_{-} \text {ferrite }}-R_{w / o_{-} \text {ferrite }}\right) / R_{w / o_{-} \text {ferrite }}\right] .
\end{aligned}
$$

Here, $\Delta L$ and $\Delta R$ are the percentage changes in the system's equivalent inductance and resistance, respectively. Also, $L_{w / o \text { ferrite }}$ and $R_{w / o \text { ferrite }}$ represent the equivalent inductance and resistance of the system [see Figs. 5(a) and 5(b)], in which ring-shaped inner ferrites are not used. Similarly, $L_{w_{f} \text { ferrite }}$ and $R_{w_{\text {fferrite }}}$ stand for the equivalent inductance and resistance of the system [see Figs. 5(c) and 5(d)], in which ring-shaped ferrites are placed inside the coils.

In the figure, it is seen that with placements of the ring shape ferrites inside the coils, the system's equivalent inductance increases by around $25 \%$ at $20 \mathrm{kHz}$ and $19 \%$ at 100 $\mathrm{kHz}$. Here, an increase in the system's inductance indicates enhancement in the flux density and coupling. Similarly, with the ring-shaped inner ferrites, the system's equivalent resistance increases by around $65 \%$ at $20 \mathrm{kHz}$ and $50 \%$ at 100 $\mathrm{kHz}$. Results show that enhancements in system's equivalent inductance and resistance values with ring-shaped inner ferrites are very similar to single and double coil arrangements.

As in single coil arrangement, we modeled and simulated the new system represented in Fig. 4 with 3D electromagnetic solver. For comparison purposes, simulations were
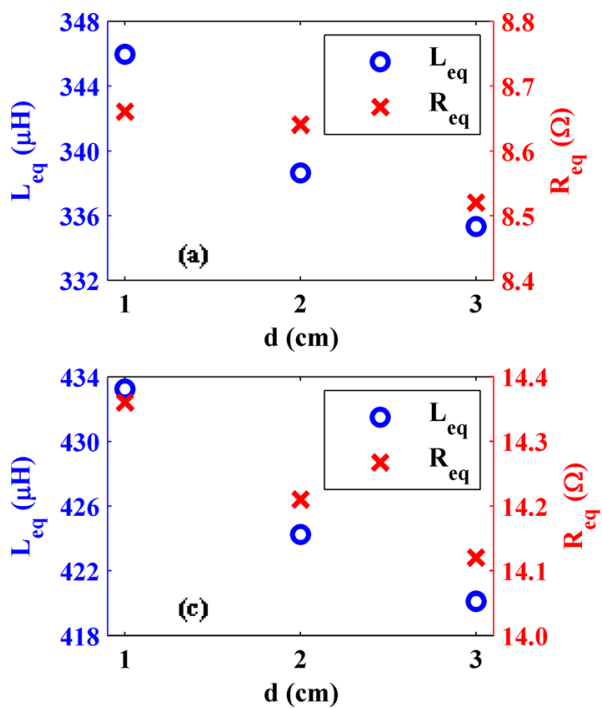
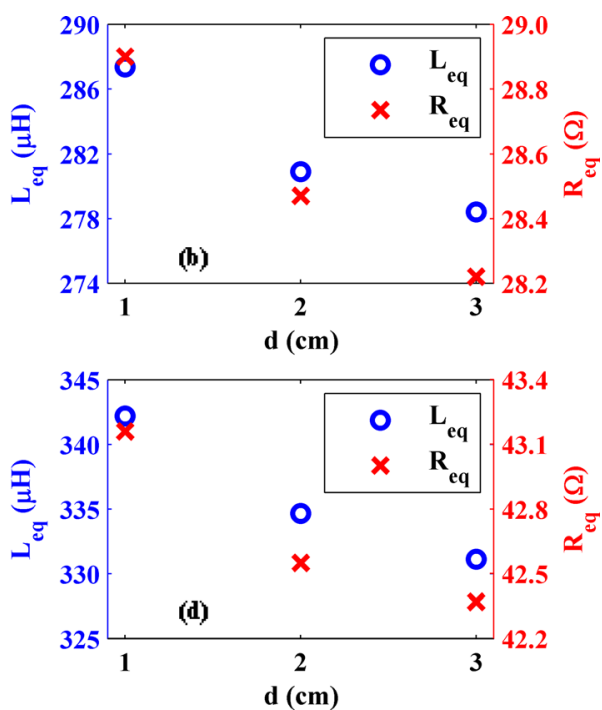

FIG. 5. Inductance and resistance of the constructed induction system (shown in Fig. 4) for various coil distances by applying $180^{\circ}$ phase differences between the coils where inner ferrites do not exist and measurements were obtained at (a) $20 \mathrm{kHz}$ and (b) $100 \mathrm{kHz}$, and inner ferrites exist and measurements were obtained at (c) $20 \mathrm{kHz}$ and (d) $100 \mathrm{kHz}$. 

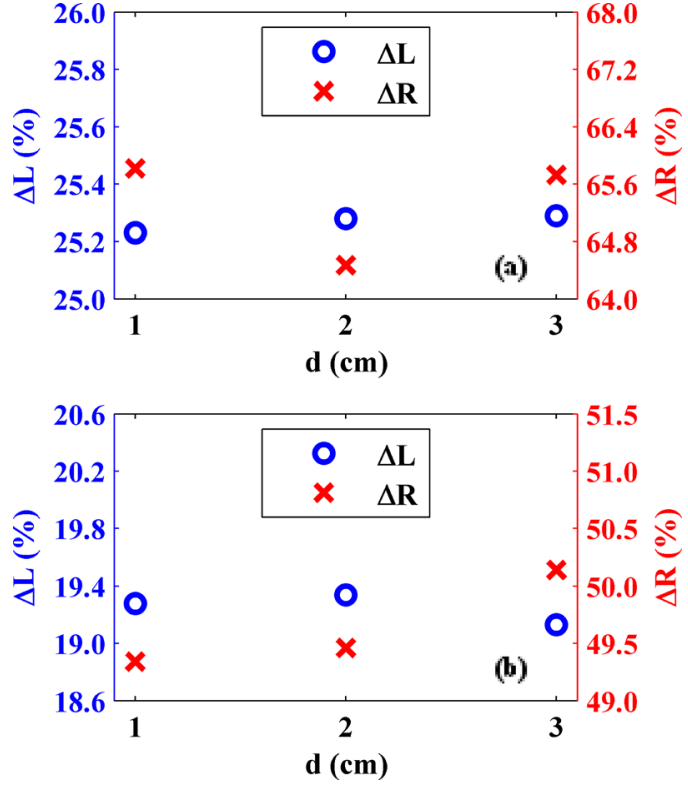

FIG. 6. Inductance and resistance changes in the system (in percentages) with ring-shaped inner ferrites placements calculated for measurements conducted at (a) $20 \mathrm{kHz}$ and (b) $100 \mathrm{kHz}$. In measurements, coils are driven with $180^{\circ}$ phase difference.

repeated with and without the ring-shaped inner ferrites. In the simulations, again mica layers were modeled as air gaps. In addition, materials used to model the parts are the same with those used to model the parts in single coil simulations such that coils were modeled to be made of copper $(\mathrm{Cu})$ material. Similarly, the ring-shaped inner ferrites and the bottom ferrite layer were modeled to be made of $\mathrm{Mn}-\mathrm{Zn}$. Also, the plate's material was selected to be AISI 430 with the electrical and magnetic parameters described earlier.

Power transfer efficiencies of the system were calculated for various distances between the coils. Results are illustrated in Fig. 7. In the figure, power transfer efficiency increases with distance between the coils when the coils are driven by $0^{\circ}$ phase difference [Figs. 7(a) and 7(b)]. On the other hand, power transfer efficiency has a decreasing trend with distance between the coils in the case of $180^{\circ}$ phase difference application [Figs. 7(c) and 7(d)]. These behaviors are very similar to inductance changes in Fig. 3 and because of destructive and constructive coupling of the coils with $0^{\circ}$ and $180^{\circ}$ phase difference applications, respectively. In addition, it is seen that the system power transfer efficiency increases from around $87 \%$ to $91 \%$ [Fig. 7(a)] and from $86 \%$ to $89 \%$ [Fig. 7(b)] in the case of $0^{\circ}$ phase difference application at $20 \mathrm{kHz}$ and $100 \mathrm{kHz}$, respectively, with the inner ferrites placement inside the coils. Similarly, ring-shaped ferrites inside the coils enable the system power transfer efficiency to increase from $88 \%$ to $92 \%$ [Fig. 7(c)] and from $86 \%$ to $89 \%$ [Fig. 7 (d)] for $180^{\circ}$ phase difference between the coils at $20 \mathrm{kHz}$ and $100 \mathrm{kHz}$, respectively.

Furthermore, we calculated the equivalent inductance of the coils in the systems and compared the results with those obtained by measurements. Similar results were found in the simulations and the measurements. For instance, the total equivalent inductance of the coils in the system without ring-shaped inner ferrites, where the coils were separated by $3 \mathrm{~cm}$ distance and $180^{\circ}$ phase difference was applied between the coils, was calculated to be $335.11 \mu \mathrm{H}$ at $20 \mathrm{kHz}$ and $255.46 \mu \mathrm{H}$ at $100 \mathrm{kHz}$. These results are very close to the inductance values represented in Figs. 5(a) and 5(b) for the same system.

\section{CONCLUSION}

In conclusion, this work presents a newly proposed coil architecture using ring-shaped inner ferrites placed in the same plane with coils. Simple proof-of-concept demonstrator systems composed of single and pair of circular coils, a ferrite layer, and a steel plate were constructed. The equivalent inductance and resistance of the model systems were comparatively measured for various coil distances with and without the ringshaped inner ferrites in place. Measurements were repeated at different frequencies and with $180^{\circ}$ phase differences between the coils in a double coil arrangement. In addition to conventional phase difference application and the ferrite layer
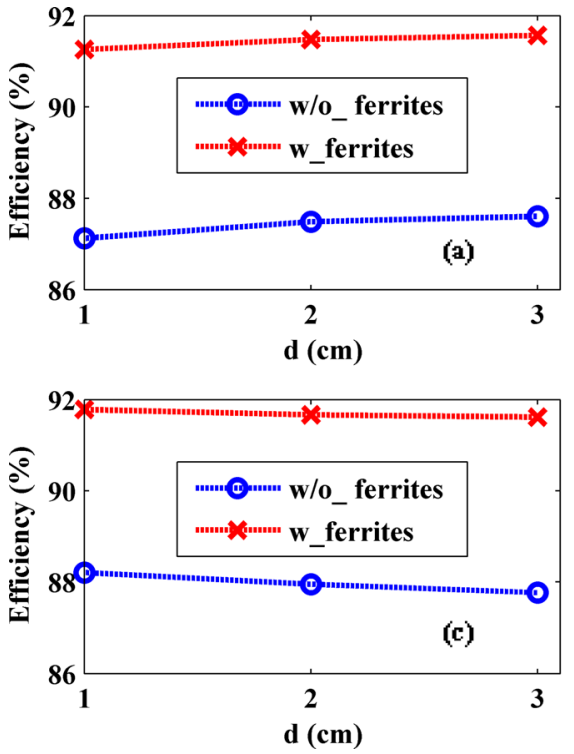
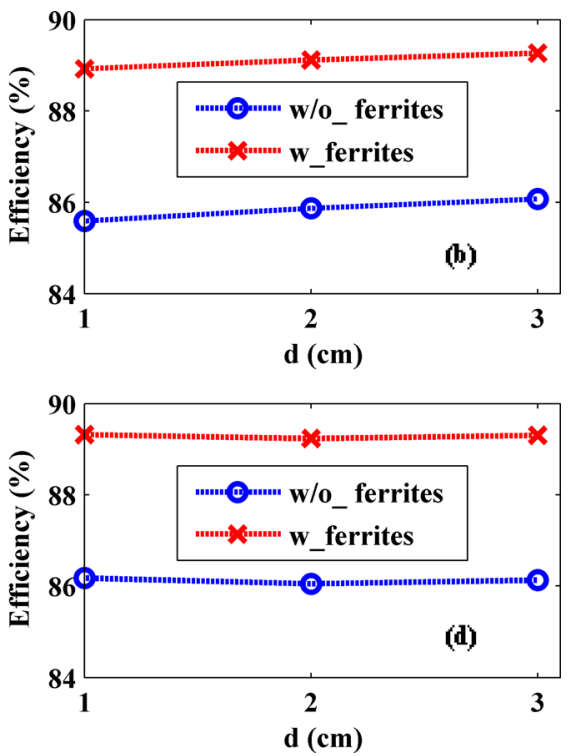

FIG. 7. Power transfer efficiency of the system (shown in Fig. 4) calculated for various coil distances with and without ring-shaped ferrites inside the coils by applying $0^{\circ}$ phase difference between the coils at (a) $20 \mathrm{kHz}$ and (b) $100 \mathrm{kHz}$ and by applying $180^{\circ}$ phase difference between the coils at (c) $20 \mathrm{kHz}$ and (d) $100 \mathrm{kHz}$. Here, labels w/o_ferrites and w_ferrites represent the systems without and with the inner ferrites, respectively. 
placement, locating ferrite inside the coils is shown to be another method for efficiency enhancement. Results indicate that with the ring-shaped inner ferrites, the total dissipated power in the system can be increased by over $65 \%$. Also, it is calculated that ring shape inner ferrites enable power transfer efficiency of the system to reach $92 \%$. The findings of this study are beneficial for inductive heating system applications where an array of small-sized coils is used. A possible extension to this work is to investigate ferrites for coupling and efficiency enhancement of coils that have 3D stacking.

\section{ACKNOWLEDGMENTS}

The authors are pleased to acknowledge the support of Arcelik A.S.

${ }^{1}$ A. Kurs, A. Karalis, R. Moffatt, J. D. Joannopoulos, P. Fisher, and M. Soljacic, Science 317, 83 (2007).

${ }^{2}$ G. Vandevoorde and R. Puers, Sens. Actuators, A 92, 305 (2001).

${ }^{3}$ T. Misawa, T. Sato, T. Takura, F. Sato, and H. Matsuki, J. Appl, Phys. 111, 07E720 (2012).

${ }^{4}$ J. G. Monroe, E. S. Vasquez, Z. S. Aspin, K. B. Walters, M. J. Berg, and S. M. Thompson, Appl. Phys. Lett. 106, 263901 (2015).

${ }^{5}$ O. Lucia, J. Acero, C. Carretero, and J. M. Burdio, IEEE Ind. Electron. Mag. 7, 35 (2013).

${ }^{6}$ J. Acero, J. M. Burdio, L. A. Barragan, D. Navarro, R. Alonso, J. R. Garcia, F. Monterde, P. Hernandez, S. Llorente, and I. Garde, IEEE Ind. Appl. Mag. 16, 39 (2010).
${ }^{7}$ J. Acero, R. Alonso, J. M. Burdio, L. A. Barragan, and S. Llorente, J. Appl. Phys. 103, 104905 (2008).

${ }^{8}$ J. Acero, C. Carretero, I. Lope, R. Alonso, and J. M. Burdio, IEEE Trans. Magn. 50, 8401107 (2014).

${ }^{9}$ K. Leidig and M. Herzog, European patent application EP 2265088 A2 (22 December 2010).

${ }^{10}$ V. T. Kilic, E. Unal, and H. V. Demir, "Space-invariant all-surface inductive heating at a constant speed enabled by tangent squircle coils in square lattice," (unpublished).

${ }^{11}$ F. Forest, S. Faucher, J.-Y. Gaspard, D. Montloup, J.-J. Huselstein, and C. Joubert, IEEE Trans. Ind. Electron. 54, 441 (2007).

${ }^{12}$ O. Lucia, J. M. Burdio, L. A. Barragan, C. Carretero, and J. Acero, IEEE Trans. Ind. Electron. 58, 5163 (2011).

${ }^{13}$ V. T. Kilic, E. Unal, and H. V. Demir, Sensors 16, 363 (2016).

${ }^{14}$ W. A. Roshen and D. E. Turcotte, IEEE Trans. Magn. 24, 3213 (1988).

${ }^{15}$ W. A. Roshen, IEEE Trans. Magn. 26, 270 (1990).

${ }^{16}$ J. Acero, C. Carretero, R. Alonso, and J. M. Burdio, IEEE Trans. Magn. 49, 1382 (2013).

${ }^{17}$ C. R. Sullivan and L. Beghou, in IEEE 14th Workshop on Control and Modeling for Power Electronics, Salt Lake City, UT, 23-26 June 2013 (IEEE, 2013), pp. 1-8.

${ }^{18}$ H.-T. Bui and S.-J. Hwang, Int. J. Heat Mass Transfer 86, 16 (2015).

${ }^{19}$ R. S. Ruffini, R. T. Ruffini, V. S. Nemkov, and R. C. Goldstein, in Proceedings of the 19th Conference in Heat Treating (1999), edited by S. J. Midea and G. D. Pfaffmann (ASM International, 2000), pp. 162-167.

${ }^{20}$ C. Carretero, J. Acero, R. Alonso, J. M. Burdio, and F. Monterde, IEEE Trans. Magn. 44, 4115 (2008).

${ }^{21}$ J. Acero, C. Carretero, O. Lucia, R. Alonso, and J. M. Burdio, IEEE Trans. Power Electron. 28, 1025 (2013).

${ }^{22}$ V. T. Kilic, E. Unal, E. Gonendik, N. Yilmaz, and H. V. Demir, IEEE Trans. Ind. Electron. 63, 7478 (2016). 